Systemic administration of either compound suppressed CIA without causing obvious immunosuppression or adverse effects. Both compounds showed similar results, which suggests that CDK4 + CDK6 are involved in pannus formation. In previous clinical anticancer trials, alvocidib induced apoptosis of malignant cells, but in CIA this drug arrested synovial fibroblasts in $\mathrm{G}_{1}$ phase without inducing apoptosis, perhaps because different CDKs are involved. Proliferating pannus cells might be more sensitive than tumor cells to the effects of CDK inhibitors, because arthritis was suppressed by only half the dose of alvocidib that would be required to exert anticancer effects. Although the novel CDK4+CDK6 inhibitor was given orally, its rapid clearance in vivo necessitated the use of fairly high doses.

The authors note that cell-cycle inhibitors suppress arthritis via different mechanisms to those of other RA therapies, so combination therapy with antirheumatic drugs plus cell-cycle inhibitors might have synergistic effects. Further investigation of this therapeutic approach is warranted.

Original article Sekine $\mathrm{C}$ et al. (2008) Successful treatment of animal models of rheumatoid arthritis with smallmolecule cyclin-dependent kinase inhibitors. J Immunol 180: 1954-1961

\section{Beneficial effects of aldosterone blockade in a mouse model of lupus nephritis}

The renin-angiotensin-aldosterone axis, which regulates renal function, is known to influence renal pathogenesis. Aldosterone is believed to have profibrotic and proinflammatory effects in the kidney, and several animal studies have shown beneficial effects of aldosterone blockade in renal dysfunction. Monrad and colleagues studied the effects of the aldosterone receptor antagonist spironolactone on the development and progression of lupus nephritis in mice.

Female NZB/NZW F ${ }_{1}$ mice, which spontaneously develop systemic lupus erythematosus, were fed spironolactone $50 \mathrm{mg} / \mathrm{kg}$, spironolactone $25 \mathrm{mg} / \mathrm{kg}$ or vehicle daily $(n=12$ for each group) from the age of 11 weeks. Half of the mice from each treatment group were killed at age 25 weeks, and the remainder were killed at age 36 weeks for kidney histopathology and gene-expression analysis.
Spironolactone treatment tended to reduce the incidence of proteinuria, although the differences between treated and control mice were not statistically significant. Compared with controls, mice in the spironolactone $25 \mathrm{mg} / \mathrm{kg}$ group had significantly lower levels of autoantibodies against single-stranded DNA $(P=0.02)$ and double-stranded DNA $(P=0.05)$ at 36 weeks. Histopathological analysis showed markedly decreased signs of inflammation and tissue damage in treated mice compared with control mice, and gene-expression analysis showed markedly decreased renal expression of several inflammatory and apoptotic genes, including those for interferon- $\gamma$, B lymphocyte stimulator and Fas (CD95) ligand.

The authors conclude that spironolactone is well tolerated and improves several parameters of lupus nephritis, and might prove effective in combination with cytotoxic agents, such as cyclophosphamide.

Original article Monrad SU et al. (2008) The role of aldosterone blockade in murine lupus nephritis. Arthritis Res Ther 10: R5

\section{A valid and sensitive patient-based disease activity score for RA}

The 28-joint Disease Activity Score (DAS28) is widely used to monitor disease activity in rheumatoid arthritis (RA); however, this assessorbased scoring method has substantial interobserver variation, which means use of DAS28 for long-term follow-up, in trials or in the clinic, is impractical. Although a patientbased disease activity score (PDAS) would avoid this disadvantage, comparability with the DAS28 would be necessary because DAS28 is used in treatment decision-making. Choy and colleagues have accordingly developed and validated a PDAS in patients with RA.

Their PDAS was based on patients' assessments of tender and swollen joints, visual analog scales for pain and general health, erythrocyte sedimentation rate (ESR) and Health Assessment Questionnaire responses. In the development cohort $(n=204)$, PDAS with ESR (PDAS1) and without ESR (PDAS2) correlated best with DAS28 $(r=0.88$ and $r=0.74$, respectively). In a separate validation cohort $(n=322)$, these measures again correlated closely with DAS28, but both were 